\title{
Looking backwards: is it time to assess veno-atrial interactions in pulmonary arterial hypertension?
}

\author{
Steven Pugliese ${ }^{1}$ and Rebecca R. Vanderpool ${ }^{2}$ \\ Affiliations: ${ }^{1}$ Pulmonary, Allergy, and Critical Care Division, University of Pennsylvania, Philadelphia, PA, USA. \\ ${ }^{2}$ Division of Translational and Regenerative Medicine, University of Arizona, Tucson, AZ, USA. \\ Correspondence: Rebecca R. Vanderpool, University of Arizona, Tucson, Translational and Regenerative \\ Medicine, 1501 North Campbell Ave, Steele Children's Research Building, 7341, Tucson, Arizona, 85724, USA. \\ E-mail: vanderpoolrrdemail.arizona.edu
}

@ERSpublications

Vena cava backflow measured using CMR imaging is a novel marker to assess veno-atrial interactions in patients with pulmonary arterial hypertension http://bit.ly/2TU4d9V

Cite this article as: Pugliese S, Vanderpool RR. Looking backwards: is it time to assess veno-atrial interactions in pulmonary arterial hypertension?. Eur Respir J 2019; 54: 1901598 [https://doi.org/10.1183/ 13993003.01598-2019].

Right ventricular (RV) function is the major determinant of mortality in patients with pulmonary arterial hypertension $(\mathrm{PAH})$. As a result, much of recent research has focused on right ventricular-pulmonary arterial coupling and, specifically, RV systolic function. As research on diastolic RV and right atrial function is beginning to emerge, the effect of the venous system on right ventricular function has been largely ignored. While observations regarding the effect of right ventricular function on venous return date to Aristotle (circa $380 \mathrm{BC}$ ), one of the earliest pathological descriptions came in 1873 when Kussmaul described a paradoxical rise in jugular venous pressure during inspiration in constrictive pericarditis [1-3]. This phenomenon can be explained by venous backflow in the setting of an elevated right atrial pressure and a noncompliant RV. To this day the determinants of venous backflow have yet to be worked out, but there is a strong clinical relevance, including the effects on RV workload and venous congestion mediated hepatic and renal dysfunction $[4,5]$.

With normal right ventricular function, ventricular contraction results in the movement of blood from the ventricle into the pulmonary circulation for oxygenation and subsequent delivery to the rest of the body (figure 1a). Invasive assessments of normal venous blood flow in dogs and healthy individuals demonstrate that the flow is remarkably pulsatile $[1,6]$. Venous blood flow to the heart is slowed during atrial systole and increases during ventricular systole [6]. Tricuspid insufficiency is one of the pathophysiological conditions that can lead to vena cava backflow. In an early study of vena cava blood flow, WeXLER et al. [6] observed marked backflow instead of forward flow during ventricular systole in a patient with tricuspid insufficiency. More contemporary evidence of this comes from cardiac magnetic resonance (CMR) imaging studies of patients with $\mathrm{PAH}$ and severe tricuspid regurgitation, where volumetric assessment of stroke volume (difference between RV end-diastolic volume and end-systolic volume) overestimates cardiac output compared to pulmonary artery and aortic flow measurements $[7,8]$. These differences were not observed in healthy volunteers or patients with mild tricuspid regurgitation suggesting that increasing tricuspid regurgitation can be a contributor to backflow into the right atrium from the RV. The contribution of right atrial contraction to venous backflow remains unknown. 
a)

Veno-atrial

interactions

\begin{tabular}{|l|l|l|l|l} 
Systemic organs $\longrightarrow-1 \begin{array}{l}\text { Vena } \\
\text { cava }\end{array}$ & $\longrightarrow-1$ & RA & RV & $\begin{array}{l}\text { Pulmonary } \\
\text { circulation }\end{array}$
\end{tabular}

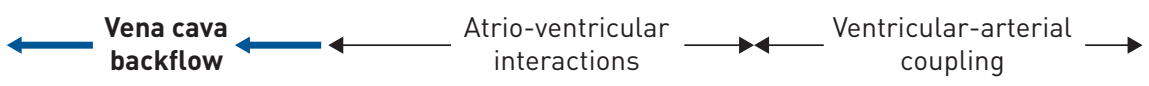

Contributing

\begin{tabular}{|c|c|c|c|}
\hline $\begin{array}{c}\text { Tricuspid valve function } \\
\text { RV diastolic stiffness } \\
\text { RA contraction } \\
\text { Venous tone/load }\end{array}$ & $\begin{array}{c}\text { Tricuspid valve function } \\
\text { RV diastolic stiffness } \\
\text { RA function? }\end{array}$ & $\begin{array}{c}\text { RV diastolic stiffness } \\
\text { Arterial afterload } \\
\text { RV contractility }\end{array}$ \\
\hline
\end{tabular}

b) Systole

$7 \%$ of vena cava backflow

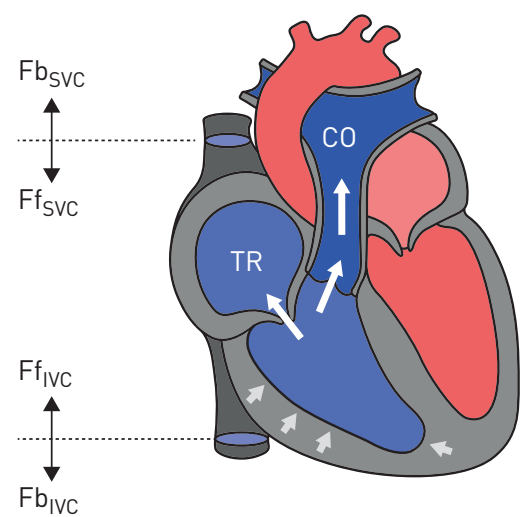

Diastole

$93 \%$ of vena cava backflow

Flow away from RA $\mathrm{Fb}=\mathrm{Fb}_{\mathrm{SVC}}+\mathrm{Fb}_{\mathrm{IVC}}$

Flow into RA

$\mathrm{Ff}=\mathrm{Ff}_{\mathrm{SVC}}+\mathrm{Ff}_{\mathrm{IVC}}$

Backward fraction

$\frac{\mathrm{Fb}}{\mathrm{Ff}}$

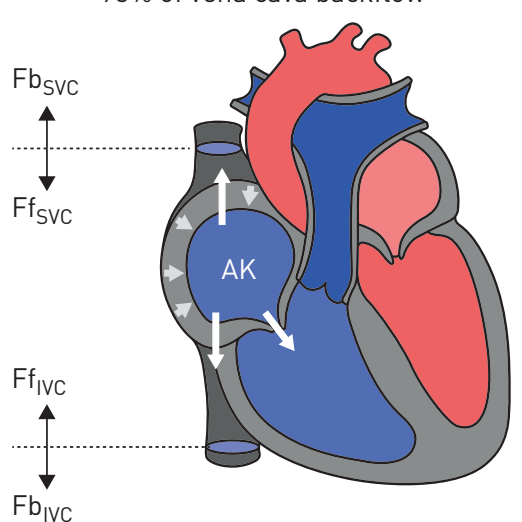

FIGURE 1 a) In the normal cardiovascular system, blood flows from the systemic organs through the superior and inferior vena cava to the right atrium (RA) and right ventricle (RV) before entering the pulmonary circulation. In the pathophysiology of pulmonary hypertension it is important to consider the factors that contribute to the ventricular-arterial coupling, atrio-ventricular interactions and finally the veno-atrial interactions. b) Backwards flow is defined as the total backwards flow (Fb) in the superior vena cava (SVC) and the inferior vena cava (IVC). Forward flow (Ff) is the total net forward flow into the right atrium from the SVC and IVC. The backward fraction is the ratio of the backflow and forward flow (Fb/Ff). Only $7 \%$ of the measured backward flow was measured during ventricular systole when there could be tricuspid regurgitation (TR). The majority of the backward flow occurred during ventricular diastole corresponding to atrial contraction/atrial kick (AK). CO: cardiac output.

In this issue of the European Respiratory Journal, MARcus et al. [9] report a detailed analysis of vena cava backflow in 62 patients with PAH. Using CMR to quantify venous flow in the superior and inferior vena cava, the authors found average backflow $(\mathrm{Fb})$ was $12 \%$ of forward flow $(\mathrm{Ff})$, and in one third of patients, backflow fraction $(\mathrm{Fb} / \mathrm{Ff}$ ) exceeded $20 \%$ (figure $1 \mathrm{~b}$ ). The maximum backflow occurred at the end of the cardiac cycle that corresponds to the atrial contraction rather than the tricuspid regurgitation. An important and novel finding of their study is that atrial contraction is the major determinant of backflow in the vena cava, accounting for $93 \%$ of total venous backflow as compared to tricuspid regurgitation. Of great clinical relevance is that backflow fraction significantly and strongly correlated with some of the best current predictors of clinical outcomes in patients with PAH, including stroke volume $(-0.61, \mathrm{p}<0.001)$, RV ejection fraction $(-0.61, \mathrm{p}<0.001)$, and RV end-systolic volume $(0.59, \mathrm{p}<0.001)$ [10, 11]. Furthermore, parameters of diastolic stiffness and right atrial function had the highest correlation to venous back flow including right atrial pressure $(0.77, \mathrm{p}<0.001), \mathrm{RV}$ end-diastolic pressure $(0.77, \mathrm{p}<0.001)$, and $\mathrm{RV}$ end-diastolic elastance $(0.65, \mathrm{p}<0.001)$.

Previous research has largely focused on cardiopulmonary interactions, including systolic RV function and the adaptation of the RV to increased afterload, while little research has focused on atrio-ventricular interactions (figure 1a) [11]. In response to increased afterload in pulmonary hypertension, the right ventricle will increase contractility to maintain the end-systolic to arterial elastance ratio (Ees/Ea) or coupling $[12,13]$. During this compensated phase, the RV has considerable reserve so that Ees/Ea and RV ejection fraction have to decrease by $50 \%$ and $>40 \%$, respectively, before there is a rapid progressive increase 
in end-diastolic volume and poor prognosis [14]. RV end-diastolic elastance or stiffness is emerging as an important clinical predictor of outcomes. An increase in RV diastolic stiffness significantly associates with increased mortality and lung transplantation in patients with pulmonary hypertension [10, 15]. A significant decrease in RV diastolic stiffness has been found in patients on 3 months of intravenous prostacyclin therapy PAH [16]. These clinical methods to assess RV diastolic stiffness rely on invasive assessments of RV diastolic pressure and the use of the single-beat method [10,15, 17], whereas venous backflow fraction can be measured non-invasively using CMR alone. Few studies have addressed the "veno-atrial" interactions but recent work investigates the role of right atrial function in PAH using echocardiography and CMR imaging [18-21]. LENG et al. [18] showed that impaired right atrial function is associated with decompensated haemodynamics and worse outcomes in patients with PAH [18]. Venous backflow allows for a complex assessment of the interaction between right atrial function, RV diastolic function, intravascular volume status and venous congestion in a single non-invasive measurement that are not provided by current gold standards of right heart failure, such as right atrial pressure and right ventricular end diastolic pressure.

Novel non-invasive metrics of RV function like venous backflow fraction have the potential to help in better understanding mechanisms of RV failure and its clinical consequences, but a few important next steps remain. Venous backflow was only measured in patients with pulmonary hypertension, so it will be important to establish venal cava flow patterns in normal subjects and define abnormal cut-off values. Furthermore, it will be imperative to determine if alterations in venous backflow correlate with established clinical outcomes of morbidity and mortality in pulmonary hypertension. Over half the patients in the study had an elevated right atrial pressure over $10 \mathrm{mmHg}$. Given the interplay between right atrial pressure, RV end-diastolic pressure, and RV stiffness, it will be important to understand whether venous backflow adds additional prognostic information in patients with a normal right atrial pressure and normal volume loading. VANDERPOOL et al. [10] recently demonstrated in a cohort of PAH patients that only stroke volume/end-systolic volume remained a significant predictor of survival after controlling for right atrial pressure, mean pulmonary artery pressure and stroke volume. Additionally, two-dimensional phase-contrast magnetic resonance imaging requires pre-defined imaging planes not typically used for CMR and requires significant technical expertise [22]. Lastly, CMR derived venous backflow fraction should be compared to Doppler ultrasound derived surrogates of venous backflow including portal vein and intrarenal vein flow assessments. Increased portal vein pulsatility can easily be measured with portable ultrasound at the bedside and observational studies suggest correlation with RV failure, and renal and hepatic dysfunction $[4,23]$.

Finally, it is important to remember that changes in the veno-atrial interactions do not cause pulmonary hypertension or right heart failure, but they have the potential to enhance our understanding and provide important markers of right heart failure. Significant vena cava backflow is potentially important in the progression of right heart failure, but additional research is necessary. The pathophysiology of RV failure is complex and better non-invasive biomarkers that associate with RV diastolic function may contribute to the development of novel treatment strategies targeting the right ventricle. As we look forward, future research studies can expand their investigations beyond systolic RV function to RV diastolic function and right atrial function in patients with $\mathrm{PAH}$ and other forms of pulmonary hypertension.

Conflict of interest: None declared.

\section{References}

1 Brecher GA. Cardiac variations in venous return studied with a new bristle flowmeter. Am J Physiol 1954; 176: 423-430.

2 Kussmaul A, Stern M. Pericarditis and the paradox pulse. Berl Klin Wochenschr 1873; 38.

3 Bilchick KC, Wise RA. Paradoxical physical findings described by Kussmaul: pulsus paradoxus and Kussmaul's sign. Lancet 2002; 359: 1940-1942.

4 Eljaiek R, Cavayas YA, Rodrigue E, et al. High postoperative portal venous flow pulsatility indicates right ventricular dysfunction and predicts complications in cardiac surgery patients. Br J Anaesth 2019; 122: 206-214.

5 Testani JM, Khera AV, St. John Sutton MG, et al. Effect of right ventricular function and venous congestion on cardiorenal interactions during the treatment of decompensated heart failure. Am J Cardiol 2010; 105: 511-516.

6 Wexler L, Bergel DH, Gabe IT, et al. Velocity of blood flow in normal human venae cavae. Circ Res 1968; 23: 349-359.

7 Hoeper MM, Tongers J, Leppert A, et al. Evaluation of right ventricular performance with a right ventricular ejection fraction thermodilution catheter and MRI in patients with pulmonary hypertension. Chest 2001; 120: 502-507.

8 Mauritz G-J, Marcus JT, Boonstra A, et al. Non-invasive stroke volume assessment in patients with pulmonary arterial hypertension: left-sided data mandatory. J Cardiovasc Magn Reson BioMed Central 2008; 10: 51.

9 Marcus JT, Westerhof BE, Groeneveldt JA, et al. Vena cava backflow and right ventricular stiffness in pulmonary arterial hypertension. Eur Respir J 2019; 54: 1900625.

10 Vanderpool RR, Pinsky MR, Naeije R, et al. RV-pulmonary arterial coupling predicts outcome in patients referred for pulmonary hypertension. Heart 2015; 101: 37-43. 
11 Vonk Noordegraaf A, Chin KM, Haddad F, et al. Pathophysiology of the right ventricle and of the pulmonary circulation in pulmonary hypertension: an update. Eur Respir J 2019; 53: 1801900.

12 Lahm T, Douglas IS, Archer SL, et al. Assessment of right ventricular function in the research setting: Knowledge gaps and pathways forward an official American thoracic society research statement. Am J Respir Crit Care Med 2018; 198: e15-e43.

13 Vonk Noordegraaf A, Westerhof BE, Westerhof N. The relationship between the right ventricle and its load in pulmonary hypertension. J Am Coll Cardiol 2017; 69: 236-243.

14 Tello K, Dalmer A, Axmann J, et al. Reserve of right ventricular-arterial coupling in the setting of chronic overload. Circ Heart Fail 2019; 12: e005512.

15 Trip P, Rain S, Handoko ML, et al. Clinical relevance of right ventricular diastolic stiffness in pulmonary hypertension. Eur Respir J 2015; 45: 1603-1612.

16 Vanderpool RR, Desai AA, Knapp SM, et al. How prostacyclin therapy improves right ventricular function in pulmonary arterial hypertension. Eur Respir J 2017; 50: 1700764.

17 Vanderpool RR, Puri R, Osorio A, et al. EXPRESS: surfing the right ventricular pressure waveform: methods to assess global, systolic and diastolic RV function from a clinical right heart catheterization. Pulm Circ 2019; in press [https://doi.org/10.1177/2045894019850993].

18 Leng $\mathrm{S}$, Dong $\mathrm{Y}, \mathrm{Wu} \mathrm{Y}$, et al. Impaired cardiovascular magnetic resonance-derived rapid semiautomated right atrial longitudinal strain is associated with decompensated hemodynamics in pulmonary arterial hypertension. Circ Cardiovasc Imaging 2019; 12: e008582.

19 Wright LM, Dwyer N, Wahi S, et al. Association with right atrial strain with right atrial pressure: an invasive validation study. Int J Cardiovasc Imaging 2018; 34: 1541-1548.

20 Alenezi F, Mandawat A, Il'Giovine ZJ, et al. Clinical utility and prognostic value of right atrial function in pulmonary hypertension. Circ Cardiovasc Imaging 2018; 11: e006984.

21 Bai Y, Yang J, Liu J, et al. Right atrial function for the prediction of prognosis in connective tissue diseaseassociated pulmonary arterial hypertension: a study with two-dimensional speckle tracking. Int $J$ Cardiovasc Imaging 2019; 35: 1637-1649.

22 Tariq U, Hsiao A, Alley M, et al. Venous and arterial flow quantification are equally accurate and precise with parallel imaging compressed sensing 4D phase contrast MRI. J Magn Reson Imaging 2013; 37: 1419-1426.

23 Beaubien-Souligny W, Benkreira A, Robillard P, et al. Alterations in portal vein flow and intrarenal venous flow are associated with acute kidney injury after cardiac surgery: a prospective observational cohort study. J Am Heart Assoc 2018; 7: e009961. 